\title{
Mental Health Consultations on College Campuses: Examining the Predictive Ability of Social Media
}

Koustuv Saha ( $\nabla$ koustuv.saha@gatech.edu )

Georgia Institute of Technology

Asra Yousuf

Georgia Institute of Technology

Ryan L. Boyd

Lancaster University

James W. Pennebaker

The University of Texas at Austin

Munmun Choudhury

Georgia Institute of Technology

\section{Research Article}

Keywords: social media, language, college students, mental health, counseling services, Reddit

Posted Date: March 9th, 2021

DOI: https://doi.org/10.21203/rs.3.rs-196605/v1

License: (c) (i) This work is licensed under a Creative Commons Attribution 4.0 International License.

Read Full License 


\title{
Mental Health Consultations on College Campuses: Examining the Predictive Ability of Social Media
}

\author{
Koustuv Saha ${ }^{*}$, Asra Yousuf ${ }^{1}$, Ryan L. Boyd ${ }^{2-4}$, James W. Pennebaker ${ }^{5}$, and Munmun \\ De Choudhury ${ }^{1}$
}

\author{
${ }^{1}$ School of Interactive Computing, Georgia Institute of Technology, Atlanta, GA, USA \\ ${ }^{2}$ Department of Psychology, Lancaster University, Lancaster, UK \\ ${ }^{3}$ Security Lancaster, Lancaster University, Lancaster, UK \\ ${ }^{4}$ Data Science Institute, Lancaster University, Lancaster, UK \\ ${ }^{5}$ Department of Psychology, University of Texas at Austin, Austin, TX, USA \\ *Corresponding Author: \\ Koustuv Saha \\ School of Interactive Computing \\ Georgia Institute of Technology \\ 756 W Peachtree St. NW \\ Atlanta, GA 30308, USA \\ koustuv.saha@gatech.edu
}

\begin{abstract}
The mental health of college students is a growing concern, and gauging the mental health needs of college students is difficult to assess in real-time and in scale. While social media has shown potential as a viable "passive sensor" of mental health, the construct validity and in-practice reliability of such computational assessments remain largely unexplored. Towards this goal, we study how assessing the mental health of college students using social media data correspond with ground-truth data of on-campus mental health consultations. For a large U.S. public university, we obtained ground-truth data of on-campus mental health consultations between 2011-2016, and collected 66,000 posts from the university's Reddit community. We adopted machine learning and natural language methodologies to measure symptomatic mental health expressions of depression, anxiety, stress, suicidal ideation, and psychosis on the social media data. Seasonal auto-regressive integrated moving average (SARIMA) models of forecasting on-campus mental health consultations showed that incorporating social media data led to predictions with $r=0.86$ and SMAPE $=13.30$, outperforming models without social media data by $41 \%$. Our language analyses revealed that social media discussions during high mental health consultations months consisted of discussions on academics and career, whereas months of low mental health consultations saliently show expressions of positive affect, collective identity, and socialization. This study reveals that social media data can improve our understanding of college students' mental health, particularly their mental health treatment needs.
\end{abstract}

Keywords: social media; language; college students; mental health; counseling services; Reddit

\section{Introduction}

Mental health on college campuses is a matter of growing concern as an increasing number of college students show rising levels of anxiety, depression, and suicidal ideation. According to the 2019 National College Health Assessment ${ }^{1} 16.7 \%$ students felt too depressed to function in the last two weeks from when the survey was conducted while $8.6 \%$ seriously considered suicide or tried to harm themselves in the past 12 months. Another decade-spanning study found that the percentage of students diagnosed with mental illness rose up from $22 \%$ in 2007 to $36 \%$ in 2017 even though the rate of treatment increased from $19 \%$ to $34 \%^{2}$. Mental health services on college campuses, including on-campus counseling centers and psychiatric clinics, therefore continuously struggle to address the increasing demands of mental health consultations in a timely fashion. A research study conducted by Penn State's Center for Collegiate Mental Health, for instance, reported a 30-40\% increase in the on-campus counseling consultations between 2009-2015, despite an only 5\% increase in enrollment ${ }^{3}$. In short, these services often lack in resources, staff, and preparedness, leading to long waiting lists and selective/infrequent consultations of many ${ }^{4}$. This understates an urgent need to meet this rise in demand of mental health services with adequate and accessible resources.

However, currently campus mental health services do not have adequate means to assess the evolving nature of demand 
or needs. While periodic surveys of students' mental health provides some barometer of mental health incidence, in terms of medication use, daily lifestyle, suicidal thoughts, depression symptoms, as well as potentially contributing academic, environmental, personal, and social factors ${ }^{5}$, they are accurate only in snapshots, and are prone to retrospective and susceptible to biases ${ }^{6}$. Since it is practically and financially unsustainable to administer surveys in real-time, when administered after a while from actual incidences, such data may not capture the time-sensitive nature of mental health needs and demands. Surveys are also difficult to scale and are limited to smaller study groups. With an increasing gap in the supply of mental health resources and their growing demand, college campuses need to find alternative means to gauge and forecast the demand of counselling services in order to cater to everyone who needs them.

To overcome such limitations, researchers and practitioners have started exploring passive sources of data, which provide dense and longitudinal behavior of individuals at scale ${ }^{7}$. Given the ubiquity and widespread use of social media, especially among the college student demographic, social media data has also been leveraged as a "passive sensor" that can act as a complementary source of unobtrusive, real-time, and naturalistic data to infer wellbeing ${ }^{8}$. Social media data is low-cost, large-scale, non-intrusive to collect, and has the potential to comprehensively reveal naturalistic patterns of mood, behavior, cognition, psychological states and social milieu, both in real-time and across longitudinal time for individuals and collectives ${ }^{9}$. Social media language consists of an individual's personal and social discourse about day-to-day concerns, and effectively reflects their health and psychosocial wellbeing in a variety of states and contexts ${ }^{10 ; 11 ; 12}$. Linguistic cues and social interactions on social media platforms have therefore, enabled researchers to study psychopathologies including depression, anxiety, stress, and loneliness ${ }^{13 ; 14 ; 15 ; 16 ; 17}$. Closely related to this research, Guntuku et al. showed how social media language distinctly associates with healthcare utilization, in terms of emergency and inpatient hospital visits ${ }^{18}$.

Social media plays a pivotal role in college students' lives, and is reflective of their behavior and psychosocial wellbeing $19 ; 20 ; 21 ; 22 ; 23$. Social media helps the college students to draw social capital benefits ${ }^{24}$, to satisfy psychosocial needs ${ }^{20}$, and seek solidarity and support during both normalcy and crisis ${ }^{21}$. Recently, researchers have studied college student wellbeing through campus-specific discussions on Reddit, which function as online analogs of offline and geographically situated college communities $^{21 ; 22 ; 25}$. Bagroy et al. measured campus-specific Mental Wellbeing Index (MWI), and found seasonal trends of mental health expressions which were higher during academic terms as compared to holidays ${ }^{22}$, and Saha et al. measured the efficacy of counseling recommendations following student deaths on college campuses 25 .

Although considerable research reveals the potential of social media data, its predictive ability in correspondence with ground-truth mental health data in colleges is yet to be validated. If validated, social media data can be appropriated to help meet the varying demands of college students' mental healthcare at various points of the academic year both in normalcy and crisis, such as the ongoing COVID-19 pandemic. This study targets this previously unexplored problem to examine if social media expressions of college students reflect mental health service utilization by college students. We conduct our study on a Reddit dataset of the online community of a large U.S. public university, which includes 66,020 posts by 18,401 unique users. We employ transfer learning classifiers to identify the language indicative of symptomatic mental health expressions of depression, anxiety, stress, suicidal ideation, and psychosis. We build seasonal auto-regressive time series models using the monthly number of mental health consultations (ground-truth) in the same university campus. We also examine the social media language using unsupervised language modeling and psycholinguistic characterization to explain the predictive ability of this data in terms of how it captures mental health concerns and challenges faced by college students. Our study bears positive implications on resource planning and management of on-campus mental health services. The ability to forecast demand of mental health services can enable on-campus health centers to plan better and cater to the students' needs during critical times.

\section{Results}

\subsection{Inferring Symptomatic Mental Health Expressions on Social Media}

We employed transfer learning classifiers to identify language indicative of depression, anxiety, stress, suicidal ideation, and psychosis on the university's subreddit posts. Out of the 66,020 total posts in 2011-2016, 23.49\% were related to depression, $21.62 \%$ to anxiety, $42.23 \%$ to stress, $14.19 \%$ to suicidal ideation, and $31.94 \%$ to psychosis. We aggregated the monthly averages of each type, and Figure 1 shows the temporal evolution of these posts. Interestingly, greatest mental health expressions occur in April, July, and November, which also somewhat coincide with examinations and stressful periods as per the academic calendars of most U.S. colleges. On the other hand, the mental health expressions are observed to be lowest in December, February, and October, which coincide with winter, spring, and fall holidays respectively.

\subsection{Associating Social Media Expressions and On-campus Mental Health Consultations}

Next, we examined if inferring the symptomatic mental health expressions bears relevance to the ground-truth data of on-campus mental health consultations. Figure 1 also shows some form of trend and seasonality in the occurrence of symptomatic mental health expressions. A Dicky-Fuller test revealed that these time series are not stationary $(p>0.05)$. Therefore, for each time 
series, we conducted trend and seasonality decomposition, and applied moving window based trend and seasonality removal to obtain transformed residual time series that passed the stationarity test $(p<0.05)$.

We conducted similar time series decomposition on our ground-truth data. Then, we obtained the cross-correlation between the residual time series of social media mental health expressions and ground-truth data of mental health visits. We built linear regression models at various lags by controlling for base-rates of the previous month's number of mental health consultations and the prevalence of mental health expressions on social media. A lag of $n$ months indicates a comparison where the social media data is shifted by $n$ months behind the ground-truth data. A higher standardized coefficient would explain a greater predictive ability of the social media expressions towards the ground-truth data. Next, Figure 2 plots and reports the cross-correlations for each of the mental health symptomatic expressions. Except for suicidal ideation, we find a positive correlation coefficient for all other measures. We find the greatest coefficient for stress (mean $e=0.38$ ), followed by depression (mean $e=0.26$ ), psychosis (mean $e=0.23$ ), and anxiety (mean $e=0.20)$; all with statistical significance $(p<0.05)$. These correlations suggest that social media data can be potentially effective to predict on-campus mental health consultations.

\subsection{Predicting On-campus Mental Health Consultations}

Now, we predict on-campus mental health consultations using seasonal auto-regressive integrated moving average (SARIMA) time series modeling. Table 1 shows the predictive performance of the two models introduced in the previous section, $M_{0}$ and $M_{1}$. We find that $M_{1}$ shows about $13 \%$ better correlation and about $41 \%$ lower error than $M_{0}$. A dependent overlapping correlation between the two model predictions shows a statistical significance $(t=-2.07, p<0.01)$. Figure 3 shows the model predictions in comparison to the actual values. Further, drawing on permutation test approaches ${ }^{26 ; 27}$, we permuted (randomized) the predictions of mental health consultations. 1,000 such permutations of randomized predictions show a Pearson's $r=0.09$ and SMAPE=32.40 at average, and a probability of 0 of better performance than either of $M_{0}$ and $M_{1}$. This rejects the null hypothesis that any prediction improvement is by chance. Overall, our results reveal that combining baseline model $\left(M_{0}\right)$ with social media based inferences of symptomatic mental health outcomes (in $M_{1}$ ) is an effective means to predict on-campus mental health consultations.

\subsection{Examining How Social Media Language Explains Mental Health Consultations}

Finally, we illuminate the characteristics of social media language that corresponds with our ground-truth. For this, we separated the social media data of the months that showed high mental health consultations (Hi-MHC) and those that showed low mental health consultations (Lo-MHC) on a median split. We conducted two types of language analysis, which we describe below.

\subsubsection{Analyzing Linguistic Cues}

First, we employed an unsupervised language modeling technique called Sparse Additive Generative Model (SAGE). Table 2 shows the most salient keywords distinctly used in Hi-MHC and Lo-MHC months. We find that the Hi-MHC months show greater prevalence of keywords related to academics and examination, such as, "finals", "hours semester", "summer classes", "textbooks", etc, and keywords related to disciplines such as, "cs majors", "geology", and "psychology", e.g., "I need urgent help. I'm about to get kicked out of my CS major. I need a 2.65 entry level GPA to advance. I made an A- in 312 and a C- in data structures, so my CS gpa at 2.66." Hi-MHC also show keywords related to "commencement" and "graduation", which could associate with the stress during post-college transition period of students ${ }^{28}$, for instance, "When my met my advisor to apply for graduation he told me that I needed a BF certificate to count as my minor, I wish I knew this before."

In contrast, Lo-MHC months show a greater prevalence of keywords related to events, such as "parties", "football", "events", "hangout", and "social", such as "The parties were pretty lame and we were bored at one. My friends and I stole some beers and broke into a pool only to get nearly arrested!" Likewise, Lo-MHC also show keywords related to people and friends. Other forms of social gatherings such as "game" and "football", and accommodations such as "frat" and "dorm" occur saliently in the Lo-MHC months, e.g., "I'm a freshman, currently pledging a frat! I like partying, programming, drinking, playing, lifting weights, and mindlessly scrolling social media for hours!."

\subsubsection{Psycholinguistic Characterization}

We next discuss the results from our psycholinguistic characterization. First, we extracted the normalized occurrences of the 50 psycholinguistic categories as per LIWC ${ }^{29}$. Then, for each category, we conducted an independent sample $t$-test between the occurrences in Hi-MHC and Lo-MHC months followed by a Benjamini-Hochberg-Yekutieli False Discovery Rate (FDR) correction. We present the results in Table 3.

Affective and Cognitive Attributes. Affective and cognitive attributes are indicative of an individual's disclosure and expressiveness in social media language. Among affective attributes, we find that Lo-MHC months show greater prevalence of affective categories, including anger, negative affect, and swear. Although all of these categories bear a negative connotation, their greater occurrence reflects greater expressiveness, which is known to be a positive wellbeing indicator ${ }^{30}$. This might associate 
with people venting out more often about their campus life, such as in, "Now I have even more reason to not live here next year. Fuck this place!" Among cognitive attributes, we find that the Hi-MHC months show a greater prevalence of tentativeness and discrepancies, which indicate an individual's insecurity and low degree of immediacy about the situation ${ }^{29 ; 31}$. In contrast, the Lo-MHC months show a greater prevalence of certainty, percept, hear, and see, e.g., "If you want to save your bandwidth, go to a computer lab, and watch youtube / listen to grooveshark / watch netflix all day long." The greater use of these category of language has been associated with an individual's better cognitive functioning and mental health ${ }^{32}$.

Linguistic Style Attributes. We first examine pronoun usage; pronouns are markers of social attention and connectedness. We find that $\mathrm{Hi}-\mathrm{MHC}$ months show a greater prevalence of first person singular and second person pronouns - these could be indicative of heightened self-attentional focus, first-hand accounts of personal events, narrative, and conversational language ${ }^{32}$, for example, "I added psychology in my second year. I have learned that this is a very rigorous path to take, a huge commitment, and that you may need to take an extra year to complete. I must have taken at least 15 semester hours for every semester I spent here, peaking at $21 \mathrm{hrs}$ last semester.", where an individual describes the challenges of their college journey. In contrast, Lo-MHC months show a greater prevalence of first person plural pronoun which associates with narrating as a collective identity ${ }^{21 ; 33}$, such as in "There are plenty of ways to socialize here, as we have several student organizations.". We also see a greater use of several function words including preposition, conjunction, relative, and inclusive in the Hi-MHC months, which are known to relate with personal narrative writing style ${ }^{33}$.

Personal and Social Concerns. Among personal and social attributes, we find that Hi-MHC show a greater usage of work and achievement keywords, which could associate with discussions on career and self-actualization, and a greater use of money may associate with students discussing financial concerns, e.g., "Is it possible to consolidate jobs, save money and improve level of service? What would be an implementation to achieve this?". Hi-MHC also show a greater use of keywords related to home, which could include challenges with roommate, e.g., "I got stuck on a top floor between a bad roommate and an old, tiny room, such a terrible year." In contrast, Lo-MHC months show a greater use of social words, such as in "I have a lot of free time and realized I really don't have a lot of friends. I've always been a social person, but it's been hard to make friends at this time of the year, since classes and clubs and everything are ending. How do you recommend I meet some new peeps?".

\section{Discussion}

This study showed that social media interactions of college students can help predict ground-truth data of on-campus mental health consultations. We adopted machine learning approaches to infer mental health expressions on a university's Reddit community, and then incorporated the model outcomes in time series forecasting models of the normalized number of on-campus mental health consultations. First, we found that (online) mental health expressions of college students correlate with (offline) mental health service utilization on college campus. Second, we found that the SARIMA model of forecasting on-campus mental health consultations accounting for social media data could predict the ground truth within $10.65 \%$ of error, which was also $38 \%$ lower error than models that did not include social media data. Finally, we conducted a deeper dive into the language of social media posts by comparing the data of months with high and low mental health visits using psycholinguistic characterization and an unsupervised language modeling called SAGE. We found that the months of high mental health visits tend to show a greater prevalence of words related to academics, academic examinations, career, and psycholinguistic attributes indicative of worse mental wellbeing, whereas the months of lower mental health visits show a greater prevalence of words related to social, partying, leisure, and psycholinguistic attributes indicative of better mental health. Together, social media data bears the ability to capture the language and social interaction of college students, and therefore can function as a "verbal sensor" to predict mental health needs and demands of college students.

This work establishes the construct validity of computational assessments of mental health from social media data. This data can therefore serve as an unobtrusive and passive lens to gauge offline critical measures that are otherwise challenging to predict, including other forms of mental health service utilization. Our study also demonstrated the face validity of this data, where it revealed discussions and concerns related to local, contextual, and contemporary events of interest, for example, during certain political event on gun laws in U.S., a student posted, "It's fucking nonsensical to carry a pistol around campus despite a handgun license!" Likewise, following a student death on the campus, students felt stressed and anxious about the event, e.g., "It is so depressing! Seems like he jumped out wanting to die." These kind of contextual and timely information about the community pulse can help college stakeholders including administrators, policymakers, and wellbeing councils to gauge the needs of the students and accordingly ensure that adequate resources are available to meet the demands of mental health related services. Additionally, campus stakeholders can hold awareness and support initiatives as a part of their campus mental health response to help students during their college stay. Together, this study bears implications in designing proactive and tailored support and crisis rehabilitation efforts on college campuses, and making resource allocation more evidence-based. 


\section{Limitations and Future Directions}

Our study has limitations, some of which also suggest novel and impactful future directions. We cannot claim clinical validity to our assessments, and building upon prior work ${ }^{34}$ is a direction to evaluate in the future. The findings of our study is limited to one college campus and a single form of ground truth data (on-campus mental health consultations). However, our computational approaches can easily be translated and adapted on other college campuses and for other wellbeing measures. We also note that social media data suffers from limitations of sparsity and self-selection, i.e., this data only allows us to measure those who use and choose to post on social media. Future work can validate mental wellbeing assessments from other social media streams that allow longitudinal posting, instantaneous interactions, and private socializations such as Facebook, Twitter, or Snapchat can provide rich and complementary information about individual and collective mental health on college campuses.

\section{Methods}

\subsection{Ground-truth Data of On-Campus Mental Health Service Utilization}

This research builds upon health center data stemming from a large public university in the southern U.S. with an enrollment of over 50,000 students. Our ground-truth dataset comprises the count of monthly health center visits by students at the same university. The visits are classified into two types: visits related to mental health issues, and those unrelated to mental health issues. This data spans a period of 84 months: September 2009 to August 2016.

For the purposes of our study, we normalized the monthly measure of mental health consultations as the percentage of enrolled students who sought mental health service in the same month. Such a normalization facilitates two goals -1 ) minimalization of confounding outliers and distortion due to total number of enrolled students; and 2) preservation of the privacy of the university and the students of the university whose data is being studied.

\subsection{Social Media Data}

We focus on the social media data pertaining to the college students from the same university. For this purpose, we use data from Reddit. Reddit is a popular social media platform among the age group between 18-29 years: Pew Research found that $65 \%$ of Reddit users are young adults ${ }^{35}$. This age group aligns with the typical college student demographic. Prior work has also used Reddit to study college students $21 ; 22 ; 25 ; 36$. Reddit facilitates focused conversations through "subreddits" that comprise of members interested in a specific topic. Many colleges have a dedicated subreddit community, which provides a common portal for the students on a campus to share and discuss about a variety of issues related to their personal, social, and academic life ${ }^{21 ; 22}$. We obtained the data from the subreddit corresponding to the same university under study, using the BigQuery API which hosts Reddit data archives ${ }^{21 ; 22}$. This archive included 66,020 posts by 18,401 unique users averaging at 33 posts per day between May 2011 and August 2016. The rest of the paper studies this period as this overlaps with our ground-truth data availability as well.

\subsection{Modeling Approach}

Our primary objective concerned examining if the online college community data is reflective of on-campus mental health service consultations. We identified the language indicative of symptomatic mental health outcomes from these social media posts. Then, we conducted time-series modeling to predict the mental health visits. We evaluated if including information gathered from social media data improved the predictions.

Measuring Symptomatic Mental Health Expressions on Social Media Data. We quantified mental health related expression in Reddit posts using machine learning classifiers identifying the language indicative of symptomatic mental health expressions of depression, anxiety, stress, suicidal ideation, and psychosis. We adopted the approach presented in Saha et al. ${ }^{15}$. Essentially, these classifiers are built using transfer learning methodologies, i.e., transferring a classifier trained on a different labeled dataset. These classifiers are $n$-gram $(n=1,2,3)$ based binary SVM models where the positive class of the training datasets come from appropriate subreddits, i.e., $r /$ depression for depression, $r /$ anxiety for anxiety, $r /$ stress for stress, $r /$ SuicideWatch for suicidal ideation, and $r / p s y c h o s i s$ for psychosis, and the negative class of training data comes from non-mental health content on Reddit - a collated sample of 20M posts, gathered from 20 subreddits from Reddit's home page such as r/AskReddit, r/aww, $r /$ movies, etc. These classifiers perform at a high accuracy of approximately 0.90 on test data ${ }^{15}$. We used the classifiers to label each post in our Reddit dataset with binary ( 0 or 1 ) labels of each symptomatic mental health expression.

Predicting Mental Health Service Utilization. To predict monthly mental health consultations, we adopted a time series modeling approach. We used seasonal auto regressive integrated moving average techniques (SARIMA) — a standard time series forecasting method based on past behavior accounting for seasonality ${ }^{37}$. We draw on $k$-fold $(k=10)$ cross-validation approach to predict and evaluate our modeling approaches. We first set aside the data from the first year of our dataset (2011) as the default training set so that the models could learn from the same baseline historical data. Then, we obtained various 
combinations of 10 -folds, i.e., $90 \%$ of the remaining data was used to build a model that predicted the monthly mental health consultations on the remaining $10 \%$ data, and we iterated on various combinations to predict the entire dataset. We pooled all the predictions together to compare against the actual values and compute the $r$, mean absolute error (MAE), and symmetric mean absolute percent error (SMAPE) values.

- Model $M_{0}$ is trained using only the time series of on-campus mental health consultation data. This model can be considered to be the one used in most in-practice purposes, or as our baseline model.

- Model $M_{1}$ is trained using the time series of on-campus mental health consultation data, in conjunction with the time series of monthly aggregated mental health discussions in social media. For this, we calculate the monthly average of posts relating to depression, anxiety, stress, and suicidal ideation as identified by our classifiers.

We used the above trained models to separately predict the number of monthly consultations in test data. We measured the prediction error between the actual and predicted data as MAE and SMAPE (computes error as a percentage metric between 0-100). For both error measures, lower values indicate lower error and better predictive performance. We calculated the Pearson's correlation $(r)$ between the actual and predicted data where higher values directly associate with better performance. If $M_{1}$ shows comparatively better predictive performance than $M_{0}$, we would conclude that using social media data contributes to better predict on-campus monthly mental health consultations. To measure statistical significance in prediction differences between $M_{0}$ and $M_{1}$, we conducted $t$-tests using the dependent overlapping correlation method, which controls for comparing against a common variable of interest (here, the ground-truth number of monthly on-campus mental health consultations) ${ }^{38}$.

\subsection{Analyzing the Social Media Language of Mental Health}

Finally, we interpreted how social media language associates with on-campus mental health consultations. We obtained the months of high and low number of mental health visits - we adopted a median split on the normalized number of visits in a month. Then, we examined the distinction of these periods as per social media language. This examination would help establish the face validity of the social media language in correspondence to the ground-truth. We conducted two analyses:

First, we adopted an unsupervised language modeling technique called the Sparse Additive Generative Model (SAGE) ${ }^{39}$. Given two documents, SAGE finds the keywords that distinguish the documents by comparing the parameters of two logistically parameterized multinomial models using a self-tuned regularization parameter that controls the tradeoff between frequent and rare terms. We aimed to obtain keywords that would relate with the key concerns faced by college students that lead to heightened mental health concerns.

Second, we conducted a psycholinguistic analysis. For this, we used the well-validated psycholinguistic lexicon, Linguistic Inquiry and Word Count (LIWC) ${ }^{29}$. LIWC characterizes social media language in 50 psycholinguistic attributes ranging across affect, cognition and perception, interpersonal focus, temporal references, lexical density and awareness, and personal and social concerns. This analysis would help to contextualize the social media language of college students in the literature on mental health and therefore explain the predictive ability of social media language.

\section{Data Availability}

The datasets analyzed during the current study are not publicly available to protect the privacy of the university campus, health center, and students whose data is being studied. The public Reddit data would be available upon request and subject to an appropriate data use agreement, if applicable.

\section{Acknowledgements}

KS and MDC were partly supported through NIH grant \#R01MH117172 to MDC.

\section{Ethics Declaration}

Because this work used publicly accessible Reddit data without any interaction with individuals whose data is analyzed, it did not qualify for ethics board approval. However, utmost care was taken to secure the privacy of the individuals in the dataset. This paper used de-identified data for analyses and presents paraphrased quotes to minimize traceability.

\section{Contributors}

KS and MDC designed the research; KS conceptualized and developed the analytic techniques; KS and AY gathered and analyzed the data; KS, AY, RB, JP, and MDC interpreted the results; KS, AY, and MDC drafted the paper; and RB and JP read, edited, and provided feedback on the paper.

\section{Competing Interests}

None declared. 


\section{References}

1. American College Health Association et al. American college health association-national college health assessment spring 2019 reference group data report (abridged): the american college health association. 2019.

2. Sarah Ketchen Lipson, Emily G Lattie, and Daniel Eisenberg. Increased rates of mental health service utilization by us college students: 10-year population-level trends (2007-2017). Psychiatric services, 70(1):60-63, 2019.

3. (CCMH) Center for Collegiate Mental Health (CCMH). 2018 annual report. 2018.

4. Robert P Gallagher. National survey of college counseling centers 2014. 2015.

5. Nuran Bayram and Nazan Bilgel. The prevalence and socio-demographic correlations of depression, anxiety and stress among a group of university students. Social psychiatry and psychiatric epidemiology, 43(8):667-672, 2008.

6. Roger Tourangeau, Lance J Rips, and Kenneth Rasinski. The psychology of survey response. Cambridge University Press, 2000.

7. Rui Wang, Fanglin Chen, Zhenyu Chen, Tianxing Li, Gabriella Harari, Stefanie Tignor, Xia Zhou, Dror Ben-Zeev, and Andrew T Campbell. Studentlife: assessing mental health, academic performance and behavioral trends of college students using smartphones. In Ubicomp.

8. Koustuv Saha, Larry Chan, Kaya De Barbaro, Gregory D Abowd, and Munmun De Choudhury. Inferring mood instability on social media by leveraging ecological momentary assessments. Proceedings of the ACM on Interactive, Mobile, Wearable and Ubiquitous Technologies, 1(3):95, 2017.

9. Scott A Golder and Michael W Macy. Diurnal and seasonal mood vary with work, sleep, and daylength across diverse cultures. Science, 333(6051):1878-1881, 2011.

10. Kokil Jaidka, Salvatore Giorgi, H Andrew Schwartz, Margaret L Kern, Lyle H Ungar, and Johannes C Eichstaedt. Estimating geographic subjective well-being from twitter: A comparison of dictionary and data-driven language methods. Proceedings of the National Academy of Sciences, 117(19):10165-10171, 2020.

11. Johannes $C$ Eichstaedt and Aaron $C$ Weidman. Tracking fluctuations in psychological states using social media language: A case study of weekly emotion. European Journal of Personality, 34(5):845-858, 2020.

12. Ryan L Boyd and James W Pennebaker. A way with words: Using language for psychological science in the modern era. Consumer psychology in a social media world, pages 222-236, 2015.

13. Munmun De Choudhury, Michael Gamon, Scott Counts, and Eric Horvitz. Predicting depression via social media. In ICWSM, 2013.

14. Glen Coppersmith, Mark Dredze, and Craig Harman. Quantifying mental health signals in twitter. In Proc. ACL CLCP Workshop, 2014.

15. Koustuv Saha, Benjamin Sugar, John Torous, Bruno Abrahao, Emre Kıcıman, and Munmun De Choudhury. A social media study on the effects of psychiatric medication use. Proceedings of the International AAAI Conference on Web and Social Media, 13(01):440-451, Jul. 2019. URL https://www. aaai.org/ojs/index.php/ICWSM/article/ view/3242.

16. Sharath Chandra Guntuku, Rachelle Schneider, Arthur Pelullo, Jami Young, Vivien Wong, Lyle Ungar, Daniel Polsky, Kevin G Volpp, and Raina Merchant. Studying expressions of loneliness in individuals using twitter: an observational study. BMJ open, 9(11), 2019.

17. H Andrew Schwartz, Johannes C Eichstaedt, Margaret L Kern, et al. Personality, gender, and age in the language of social media: The open-vocabulary approach. PloS one, 8(9):e73791, 2013.

18. Sharath Chandra Guntuku, H Andrew Schwartz, Adarsh Kashyap, Jessica S Gaulton, Daniel C Stokes, David A Asch, Lyle H Ungar, and Raina M Merchant. Variability in language used on social media prior to hospital visits. Scientific reports, 10(1):1-9, 2020.

19. Megan A Moreno, Lauren A Jelenchick, Katie G Egan, Elizabeth Cox, Henry Young, Kerry E Gannon, and Tara Becker. Feeling bad on facebook: depression disclosures by college students on a social networking site. Depress. Anxiety, 2011. 
20. Adriana M Manago, Tamara Taylor, and Patricia M Greenfield. Me and my 400 friends: the anatomy of college students' facebook networks, their communication patterns, and well-being. Developmental psychology, 2012.

21. Koustuv Saha and Munmun De Choudhury. Modeling stress with social media around incidents of gun violence on college campuses. Proc. ACM Hum.-Comput. Interact., 1(CSCW), December 2017. doi: 10.1145/3134727. URL https://doi.org/10.1145/3134727.

22. Shrey Bagroy, Ponnurangam Kumaraguru, and Munmun De Choudhury. A social media based index of mental well-being in college campuses. In Proceedings of the 2017 CHI Conference on Human Factors in Computing Systems, 2017.

23. Yiran Wang, Melissa Niiya, Gloria Mark, Stephanie M Reich, and Mark Warschauer. Coming of age (digitally): An ecological view of social media use among college students. In Proceedings of the 18th ACM Conference on Computer Supported Cooperative Work \& Social Computing, pages 571-582. ACM, 2015.

24. Nicole B Ellison, Charles Steinfield, and Cliff Lampe. The benefits of facebook "friends": Social capital and college studentsúse of online social network sites. Journal of Computer-Mediated Communication, 12(4):1143-1168, 2007.

25. Koustuv Saha, Ingmar Weber, and Munmun De Choudhury. A social media based examination of the effects of counseling recommendations after student deaths on college campuses. In Twelfth International AAAI Conference on Web and Social Media, 2018.

26. Koustuv Saha, Manikanta D Reddy, Vedant Das Swain, Julie M Gregg, Ted Grover, Suwen Lin, Gonzalo J Martinez, Stephen M Mattingly, et al. Imputing Missing Social Media Data Stream in Multisensor Studies of Human Behavior. In Proceedings of International Conference on Affective Computing and Intelligent Interaction (ACII 2019), 2019.

27. Aris Anagnostopoulos, Ravi Kumar, and Mohammad Mahdian. Influence and correlation in social networks. In Proceedings of the 14th ACM SIGKDD international conference on Knowledge discovery and data mining, pages 7-15. ACM, 2008.

28. Jude P Mikal, Ronald E Rice, Audrey Abeyta, and Jenica DeVilbiss. Transition, stress and computer-mediated social support. Computers in Human Behavior, 29(5):A40-A53, 2013.

29. Yla R Tausczik and James W Pennebaker. The psychological meaning of words: Liwc and computerized text analysis methods. Journal of language and social psychology, 29(1):24-54, 2010.

30. James W Pennebaker and Cindy K Chung. Expressive writing, emotional upheavals, and health. Handbook of health psychology, pages 263-284, 2007.

31. James W Pennebaker and Laura A King. Linguistic styles: language use as an individual difference. Journal of personality and social psychology, 77(6):1296, 1999.

32. James W Pennebaker, Matthias R Mehl, and Kate G Niederhoffer. Psychological aspects of natural language use: Our words, our selves. Annual review of psychology, 54(1):547-577, 2003.

33. Michael A Cohn, Matthias R Mehl, and James W Pennebaker. Linguistic markers of psychological change surrounding september 11, 2001. Psychological science, 15(10):687-693, 2004.

34. Michael L Birnbaum, Sindhu Kiranmai Ernala, Asra F Rizvi, Munmun De Choudhury, and John M Kane. A collaborative approach to identifying social media markers of schizophrenia by employing machine learning and clinical appraisals. Journal of medical Internet research, 19(8), 2017.

35. Pew. pewinternet.org/fact-sheet/social-media, 2018.

36. Koustuv Saha, Eshwar Chandrasekharan, and Munmun De Choudhury. Prevalence and psychological effects of hateful speech in online college communities. In Proceedings of the 10th ACM Conference on Web Science, pages 255-264, 2019.

37. Flávio Fonseca Nobre, Ana Beatriz Soares Monteiro, Paulo Roberto Telles, and G David Williamson. Dynamic linear model and sarima: a comparison of their forecasting performance in epidemiology. Statistics in medicine, 20(20):3051-3069, 2001.

38. Olive Jean Dunn and Virginia Clark. Comparison of tests of the equality of dependent correlation coefficients. Journal of the American Statistical Association, 66(336):904-908, 1971.

39. Jacob Eisenstein, Amr Ahmed, and Eric P Xing. Sparse additive generative models of text. In ICML, 2011. 


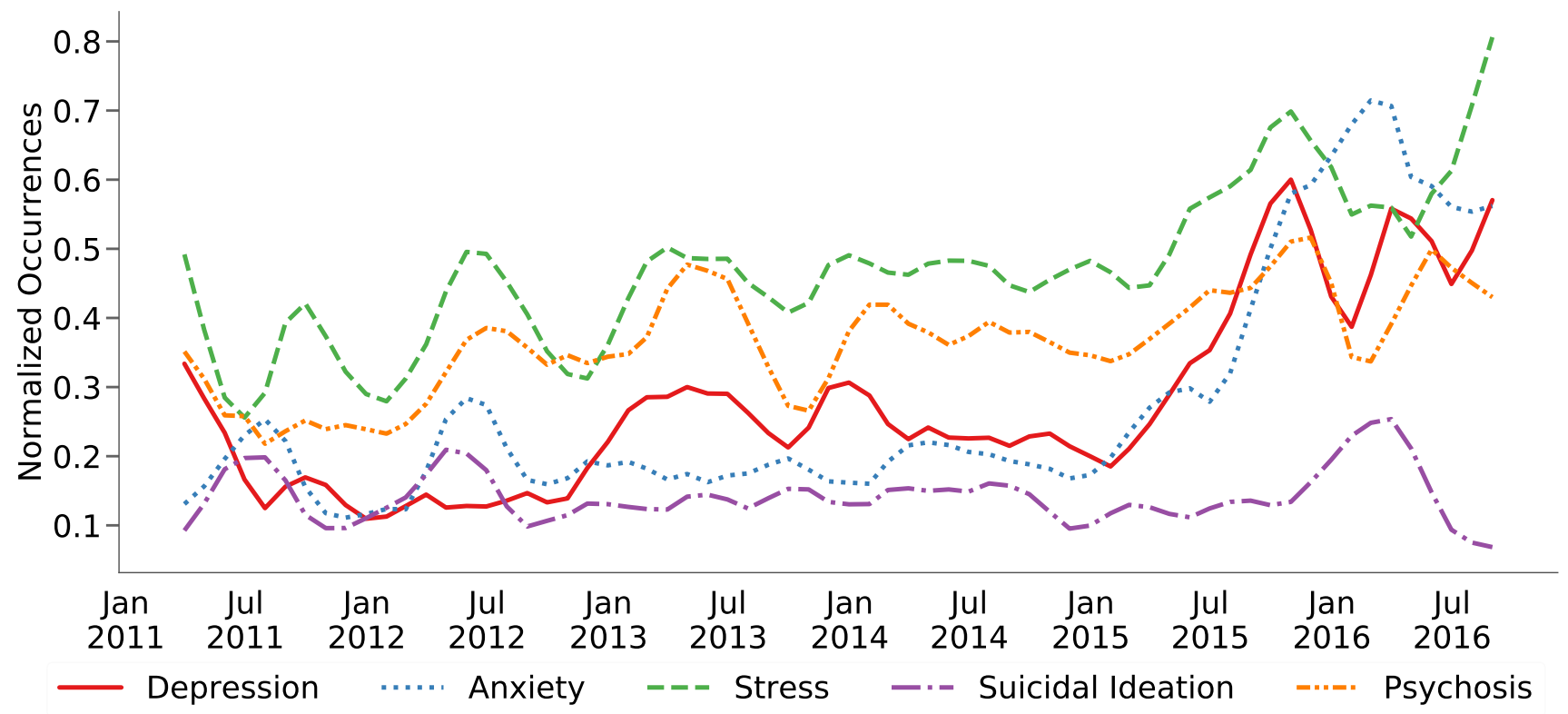

Figure 1. Temporal evolution of the normalized prevalence of social media expressions indicative of mental health symptomatic outcomes on the college subreddit. 

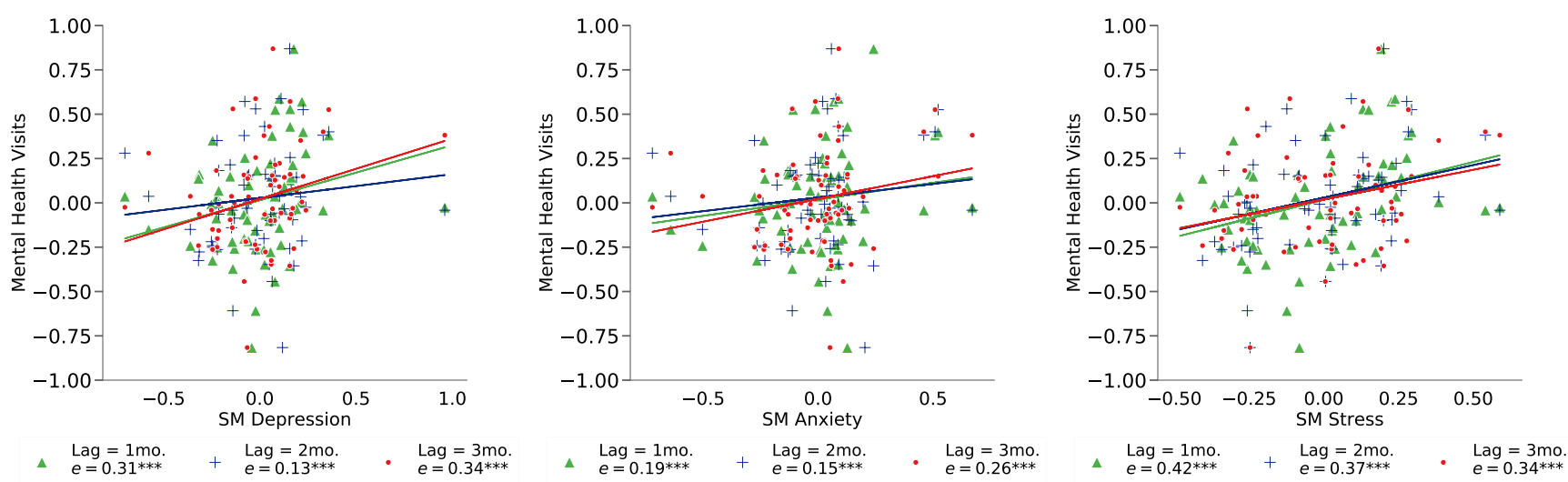

(a) Depression

(b) Anxiety

(c) Stress

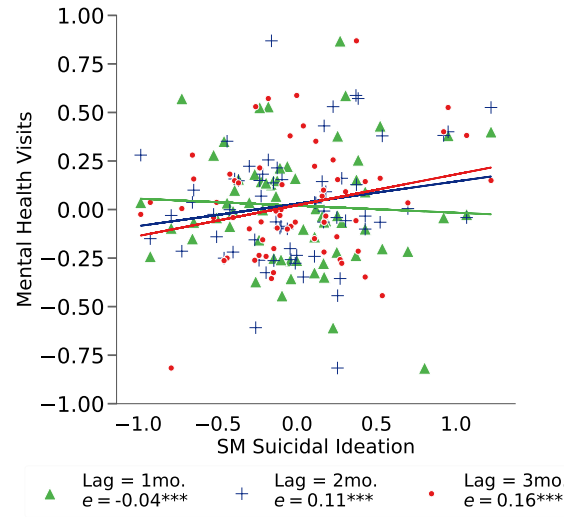

(d) Suicidal Ideation

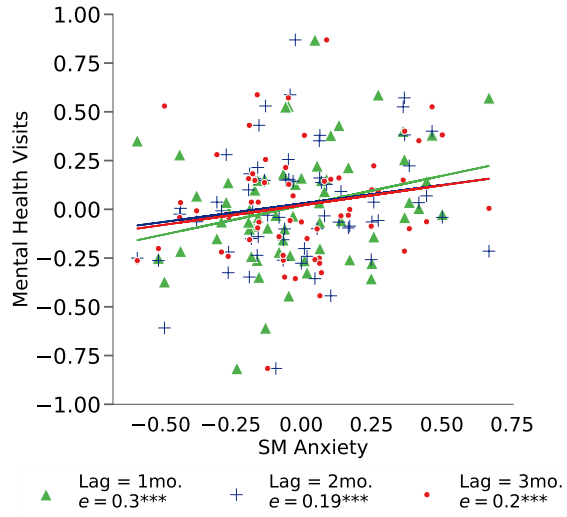

(e) Psychosis

Figure 2. Lagged Cross-correlation between the trend-seasonality removed residuals of mental health visits and mental health symptomatic expressions on social media (SM). 
Table 1. Seasonal Auto Regressive Integrated Moving Average (SARIMA) models of predicting normalized number of mental health consultations in the test dataset (year 2016 data) $(* p<0.01, * * p<0.001, * * * p<0.0001)$.

\begin{tabular}{|c|c|c|c|}
\hline Model & Pearson's $\mathrm{r}$ & MAE & SMAPE \\
\hline Model $M_{0}$ & $0.76^{\star * *}$ & 2.51 & 22.64 \\
\hline Model $M_{1}$ & $0.86^{* * *}$ & 1.96 & 13.30 \\
\hline \multicolumn{4}{|c|}{ Dependent Overlapping Correlation: $t=-2.07^{\star *}$} \\
\hline
\end{tabular}




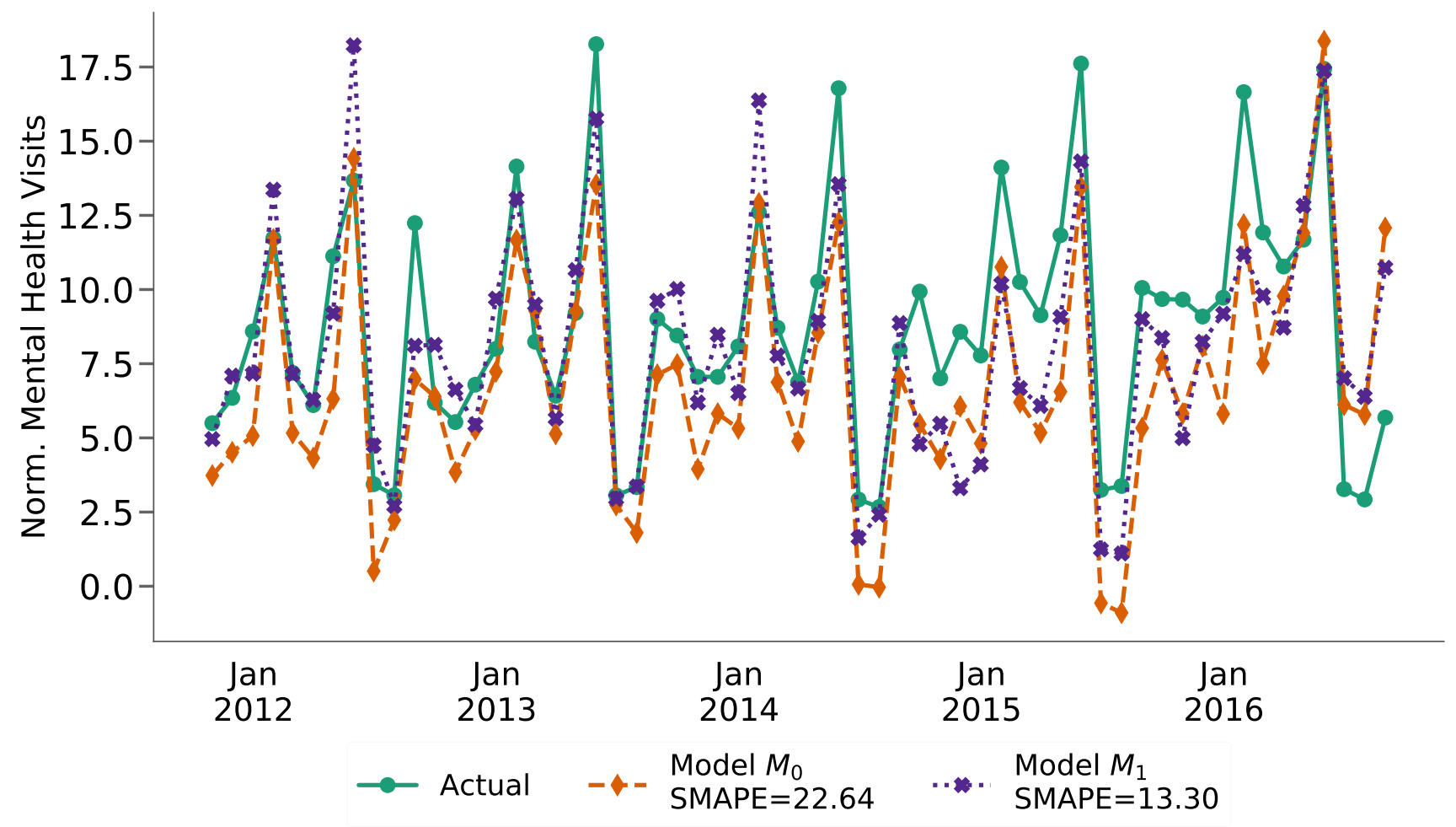

Figure 3. SARIMA models to predict the number of mental health service utilization. 
Table 2. Top salient $n$-grams $(n=1,2,3)$ distinguishing months of high and low mental health visits as per SAGE ${ }^{39}$. Bar lengths and color indicate magnitude and sign of SAGE value; pink bars (positive SAGE) indicate a saliency in Hi-MHC months whereas green bars (negative SAGE) indicate a saliency in Lo-MHC months.

\begin{tabular}{|c|c|c|c|}
\hline \multicolumn{4}{|c|}{ High Mental Health Visit Months (Hi-MHC) } \\
\hline$n$-gram & SAGE & $n$-gram & SAGE \\
\hline commencement & 2.51 & grade & 0.65 \\
\hline sword & 2.02 & gender & 0.63 \\
\hline smokers & 1.48 & mom & 0.63 \\
\hline startup & 1.14 & entry level & 0.61 \\
\hline swim & 1.08 & summer classes & 0.61 \\
\hline mass & 1.04 & equivalent & 0.59 \\
\hline april & 0.98 & drug & 0.59 \\
\hline counselors & 0.91 & dance & 0.57 \\
\hline blackboard & 0.89 & completed & 0.56 \\
\hline smell & 0.88 & summer & 0.55 \\
\hline graduation & 0.87 & comfortable & 0.55 \\
\hline shooting & 0.85 & laws & 0.53 \\
\hline finals & 0.85 & books & 0.52 \\
\hline psychology & 0.83 & swimming & 0.52 \\
\hline engineering physics & 0.82 & professional & 0.52 \\
\hline driver & 0.77 & geology & $=0.51$ \\
\hline permit & 0.77 & metro & 0.51 \\
\hline midland & 0.75 & final & 0.50 \\
\hline cs majors & 0.74 & textbooks & 0.49 \\
\hline lower division & 0.74 & labs & 0.48 \\
\hline february & 0.73 & aerospace & 0.48 \\
\hline appeal & 0.71 & exchange & 0.48 \\
\hline elective & 0.69 & gay & 0.47 \\
\hline busy & 0.68 & astronomy & 0.47 \\
\hline hours semester & 0.68 & textbook & 0.47 \\
\hline phy & 0.65 & residency & 0.47 \\
\hline
\end{tabular}

\begin{tabular}{|c|c|c|c|}
\hline \multicolumn{4}{|c|}{ Low Mental Health Visit Months (Lo-MHC) } \\
\hline$n$-gram & SAGE & $n$-gram & SAGE \\
\hline software & -0.78 & bring & -0.38 \\
\hline parties & -0.63 & laptop & -0.38 \\
\hline greek & -0.57 & buildings & -0.37 \\
\hline football & -0.57 & project & -0.37 \\
\hline internet & -0.57 & hangout & -0.37 \\
\hline season & -0.57 & funny & -0.36 \\
\hline make friends & -0.56 & union & -0.36 \\
\hline game & -0.56 & weird & -0.36 \\
\hline choice & -0.54 & ticket & -0.36 \\
\hline downtown & -0.53 & freshmen & -0.35 \\
\hline weekend & -0.52 & agree & -0.34 \\
\hline quiet & -0.47 & games & -0.34 \\
\hline phone & -0.47 & floor & -0.31 \\
\hline act & -0.46 & living campus & -0.31 \\
\hline hell & -0.46 & hall & -0.30 \\
\hline events & -0.46 & gü & -0.29 \\
\hline frat & -0.44 & score & -0.29 \\
\hline god & -0.43 & lol & -0.27 \\
\hline tech & -0.42 & world & -0.27 \\
\hline car & -0.42 & smart & -0.27 \\
\hline reasons & -0.39 & tickets & | -0.26 \\
\hline action & -0.39 & opinion & -0.26 \\
\hline teach & -0.39 & culture & -0.25 \\
\hline dorms & -0.39 & reason & | -0.25 \\
\hline bikes & -0.39 & friends & -0.25 \\
\hline party & -0.38 & social & | -0.19 \\
\hline
\end{tabular}


Table 3. Comparing psycholinguistic attributes across clusters, with mean normalized percentage occurrence in $\mathrm{Hi}-\mathrm{MHC}$ and Lo-MHC months and $t$-test statistic. Statistical significance reported after Benjamini-Hochberg- Yekutieli False Discovery Rate correction $(* * * p<.001, * * .001<p<.01, * .01<p<.05)$. Bar length and color indicates magnitude and sign of $t$-statistics. Pink bars (positive $t$ ) indicate greater prevalence in Hi-MHC months and green bars (negative $t$ ) indicate a greater prevalence in Lo-MHC months.

\begin{tabular}{|c|c|c|c|c|}
\hline \multirow[b]{2}{*}{ Category } & \multicolumn{2}{|c|}{ Hi-MHC Lo-MHC } & \multirow[b]{2}{*}{ t-stat. } & \multirow[b]{2}{*}{$\mathbf{p}$} \\
\hline & Mean\% & Mean\% & & \\
\hline \multicolumn{5}{|l|}{ Affect } \\
\hline Negative Affect & 0.44 & 0.50 & & $-3.01^{\star *}$ \\
\hline Swear & 0.28 & 0.40 & & $-5.00^{* * *}$ \\
\hline Anger & 0.46 & 0.65 & & $-6.38 * * *$ \\
\hline \multicolumn{5}{|c|}{ Cognition and Perception } \\
\hline Tentativeness & 2.84 & 2.74 & & $12.60 * * *$ \\
\hline Discrepancies & 1.48 & 1.43 & & $1.29 *$ \\
\hline Hear & 0.38 & 0.43 & $\mathbf{a}$ & $-1.62 *$ \\
\hline Certainty & 1.03 & 1.10 & & $-2.04 *$ \\
\hline Percept & 1.35 & 1.45 & & -2.64 * \\
\hline See & 0.61 & 0.67 & & $-2.71 *$ \\
\hline \multicolumn{5}{|c|}{ Interpersonal Focus } \\
\hline 2nd P. & 1.65 & 1.57 & & $1.78^{*}$ \\
\hline 1st P. Singular & 3.08 & 3.00 & 口 & $1.05 *$ \\
\hline Indef. Pronoun & 4.40 & 4.56 & & $-2.77^{*}$ \\
\hline 1st P. Plural & 0.29 & 0.33 & & -3.06 ** \\
\hline 3rd P. & 0.28 & 0.38 & & $-5.60 * * *$ \\
\hline
\end{tabular}

\begin{tabular}{|c|c|c|c|c|c|}
\hline \multirow[b]{2}{*}{ Category } & \multicolumn{2}{|c|}{ Hi-MHC Lo-MHC } & \multirow[b]{2}{*}{ t-stat. } & \multirow{2}{*}{\multicolumn{2}{|c|}{$\mathbf{p}$}} \\
\hline & Mean\% & Mean\% & & & \\
\hline \multicolumn{6}{|c|}{$\overline{\text { Lexical Density and Awareness }}$} \\
\hline Preposition & 9.44 & 9.13 & 口 & 4.69 & ** \\
\hline Relative & 10.21 & 9.95 & & $2.98 *$ & ** \\
\hline Conjunction & 4.18 & 4.06 & & $2.64 *$ & * \\
\hline Inclusive & 2.65 & 2.58 & $\square$ & $2.18 *$ & \\
\hline Quantifier & 1.73 & 1.67 & ש & $1.32 *$ & * \\
\hline \multicolumn{6}{|c|}{ Personal and Social Concerns } \\
\hline Work & 4.59 & 3.92 & & 10.26 * & *** \\
\hline Achievement & 1.26 & 1.15 & 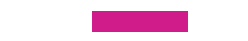 & 3.45 * & *** \\
\hline Money & 0.87 & 0.80 & & 2.56 * & \\
\hline Home & 0.58 & 0.52 & & 2.27 * & \\
\hline Social & 6.15 & 6.46 & $\mathbf{m}$ & -1.06 * & \\
\hline Religion & 0.08 & 0.11 & Q & -1.62 * & \\
\hline Bio & 0.97 & 1.04 & - & $-1.92 *$ & \\
\hline Humans & 0.52 & 0.58 & & -2.72 * & \\
\hline Sexual & 0.23 & 0.30 & & $-3.63 *$ & ** \\
\hline Body & 0.23 & 0.30 & & $-4.58 *$ & *** \\
\hline
\end{tabular}


Figures

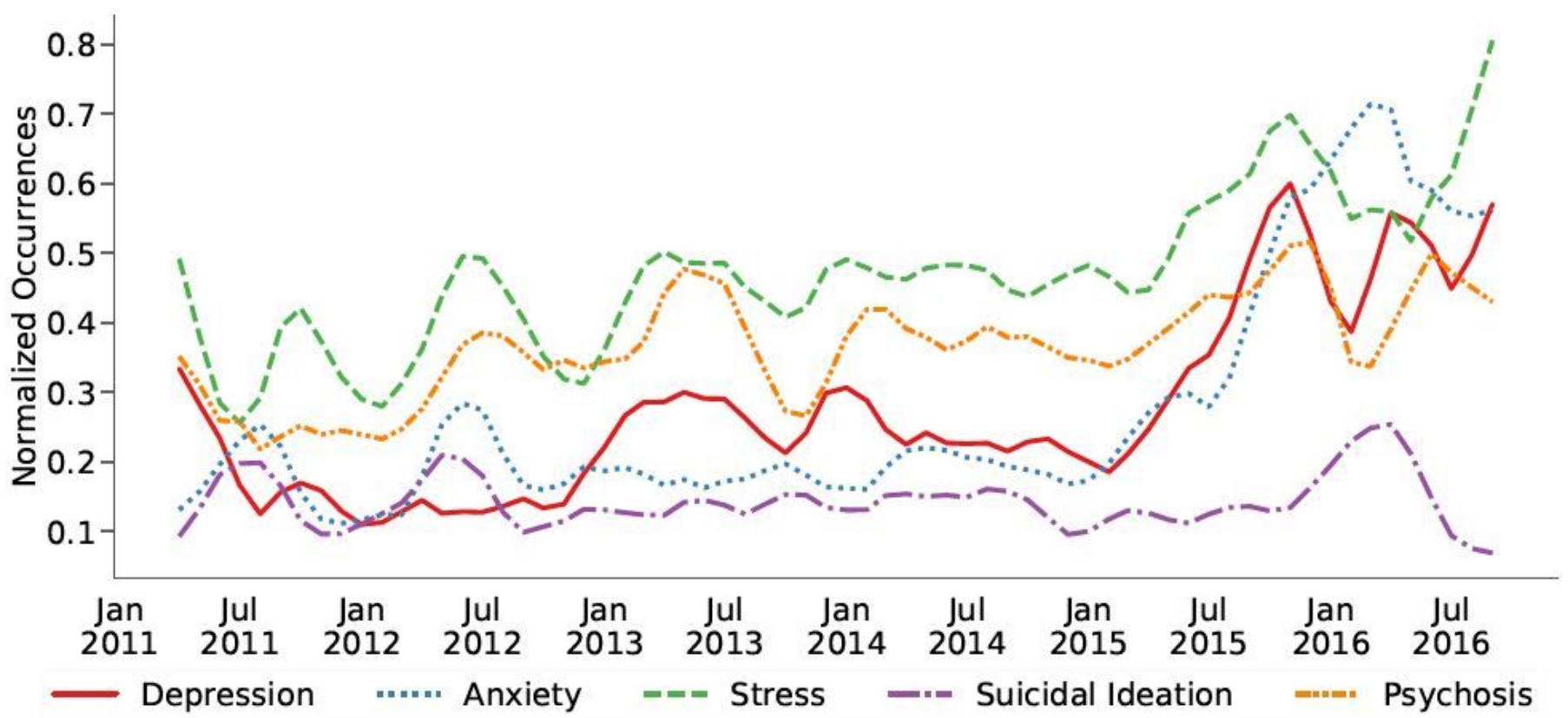

\section{Figure 1}

Temporal evolution of the normalized prevalence of social media expressions indicative of mental health symptomatic outcomes on the college subreddit. 


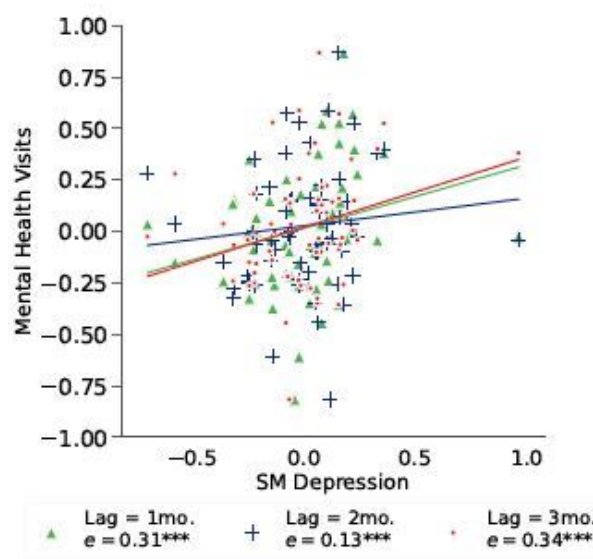

(a) Depression

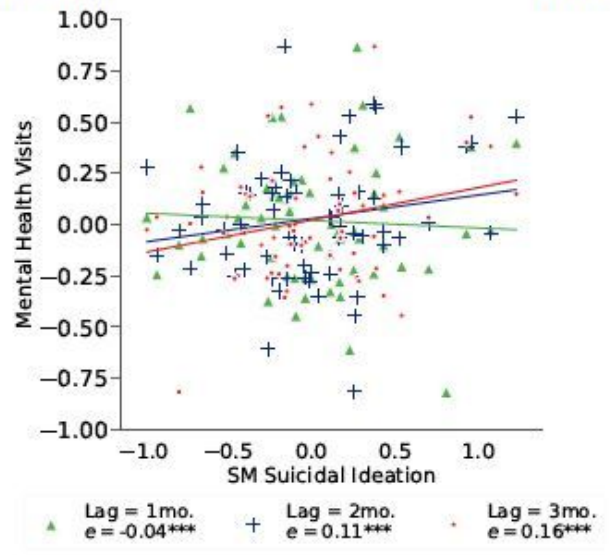

(d) Suicidal Ideation

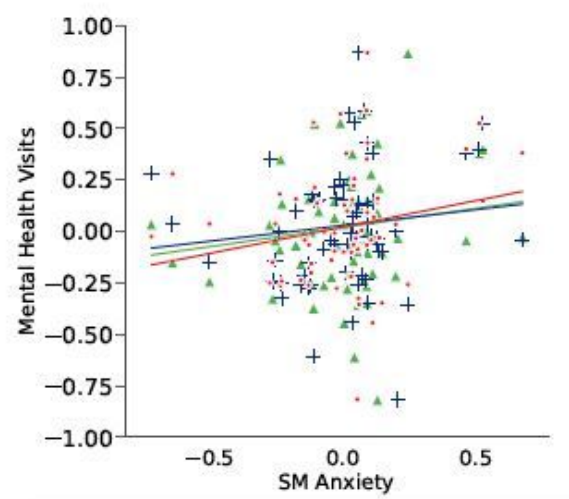

Lag $=2 \mathrm{mo}$.
$e=0.15^{* * *}$

(b) Anxiety

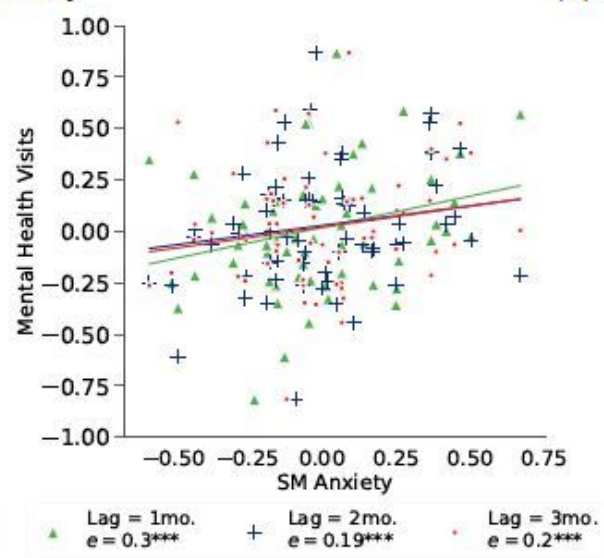

(e) Psychosis

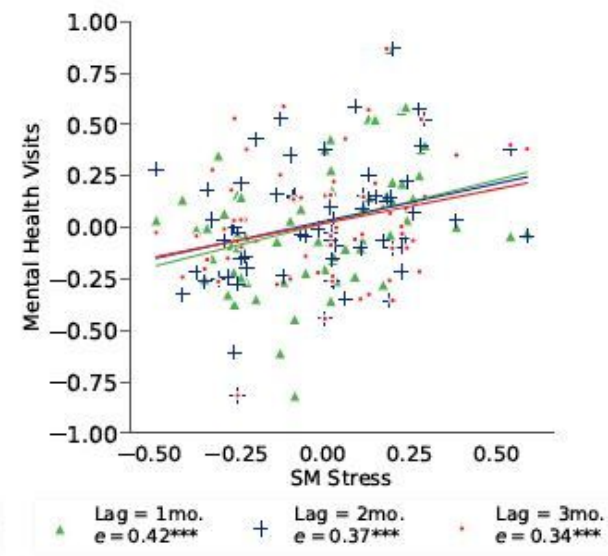

(c) Stress

\section{Figure 2}

Lagged Cross-correlation between the trend-seasonality removed residuals of mental health visits and mental health symptomatic expressions on social media (SM). 


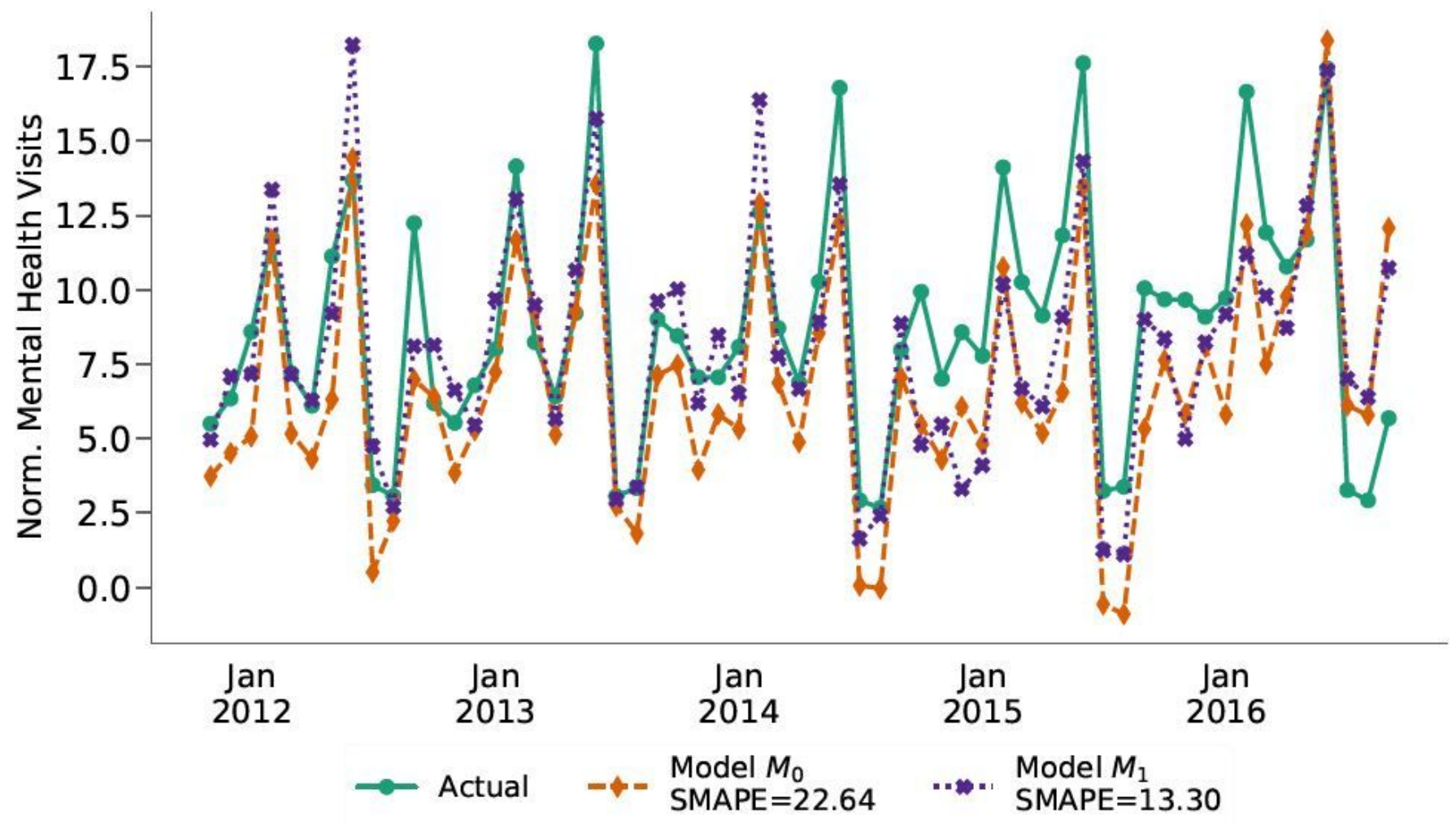

Figure 3

SARIMA models to predict the number of mental health service utilization. 